\title{
Selective Deletion of a Cell Cycle Checkpoint Kinase (ATR) Reduces Neurogenesis and Alters Responses in Rodent Models of Behavioral Affect
}

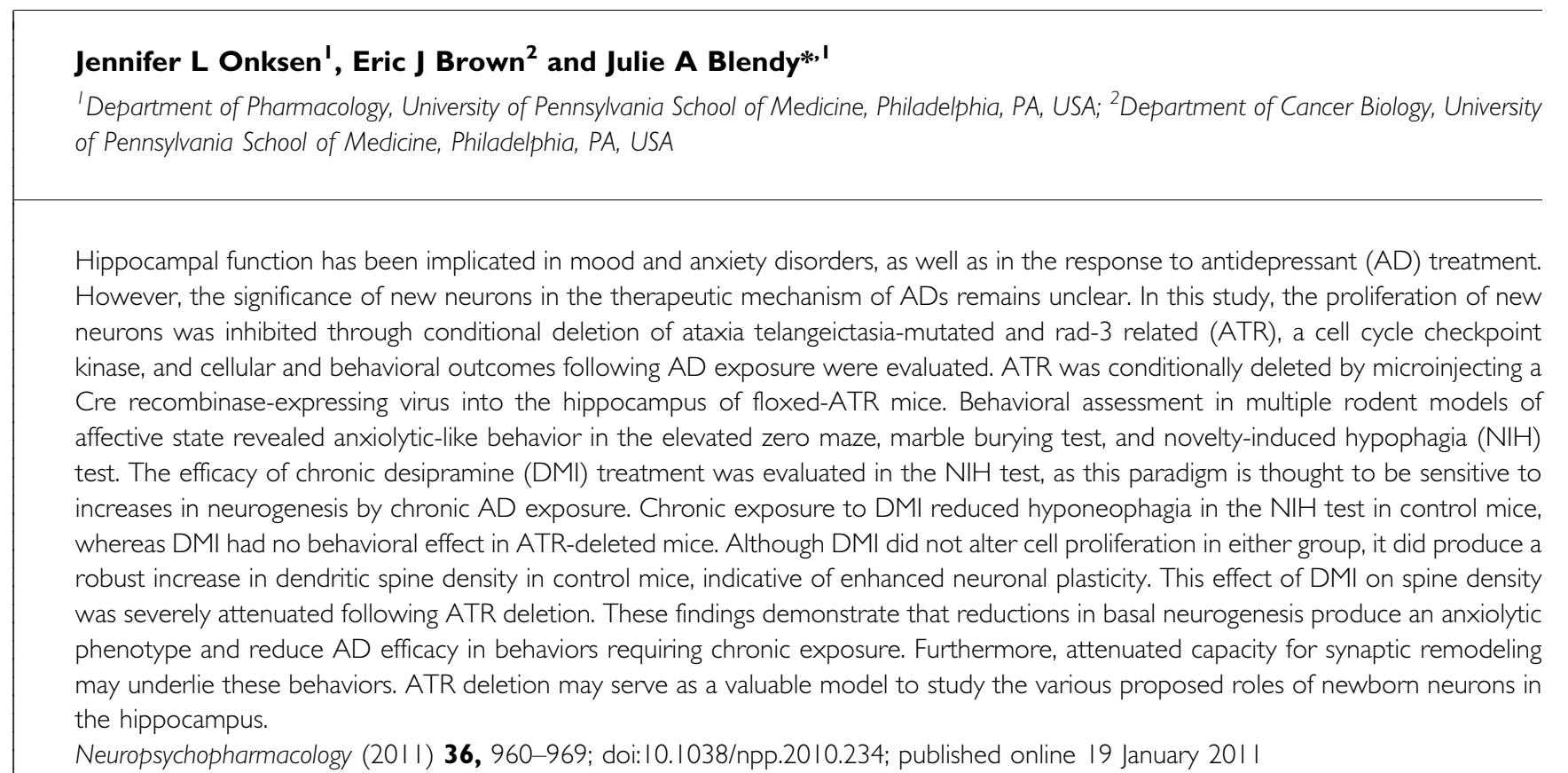

Keywords: depression; anxiety; neurogenesis; plasticity; desipramine; hyponeophagia

\section{INTRODUCTION}

Major depression is a highly prevalent psychiatric disorder characterized by a diverse manifestation and etiology. Depression and anxiety are highly co-morbid and pervasive (Merikangas et al, 2003). The ability of chronic antidepressant (AD) treatment to ameliorate the symptoms of both depression and anxiety (Schoevers et al, 2008) raises the possibility that they may share common molecular underpinnings. Hippocampal neurogenesis has been implicated in the therapeutic efficacy of AD treatment. This theory is supported by evidence that the maturation and functional integration of new neurons occurs over a period of time similar to that required for ADs to exert their therapeutic

*Correspondence: Dr JA Blendy, Department of Pharmacology, University of Pennsylvania School of Medicine, Philadelphia, PA 19104, USA, Tel: + 215898 0730, Fax: + 215573 2041,

E-mail: blendy@mail.med.upenn.edu

Received 7 August 2010; revised 2 November 2010; accepted I December 2010 effects (Malberg et al, 2000; Jessberger and Kempermann, 2003). However, the significance of new hippocampal neurons for the onset of depressive-like symptoms remains unclear (Balu and Lucki, 2009, for review). Although rodent models of chronic stress can reduce neurogenesis (Malberg and Duman, 2003; Murray et al, 2008; Elizalde et al, 2010) and induce anhedonia (Papp et al, 1991), reduced neurogenesis alone is not sufficient to induce depression and is more likely a risk factor contributing to the onset of depressive-like symptoms (Sahay and Hen, 2007).

Despite early promising reports, the relevance of new neurons to AD effects is still unclear. Fluoxetine (FLX) was shown to be ineffective in a behavioral model of AD efficacy following ablation of hippocampal neurogenesis by X-ray irradiation (Santarelli et al, 2003). Additionally, in a comparison of the inbred mouse strains $\mathrm{MRL} / \mathrm{MjP}$ and C57BL/6, Balu et al (2009) found behavioral efficacy following FLX and desipramine (DMI) treatment only in the strain that exhibited a positive neurogenic response to these drugs. In contrast, there is evidence that $\mathrm{AD}$ 
treatment elicits behavioral changes independent of hippocampal neurogenesis (David et al, 2007; Holick et al, 2008; Huang et al, 2008). Furthermore, numerous studies implicate enhanced neuroplasticity and synaptic function in the therapeutic efficacy of ADs (Bessa et al, 2009; Calabrese et al, 2009, for review; O'Leary et al, 2009; Marchetti et al, 2010).

Although depression and anxiety present with high comorbidity, little work has been done to examine neurogenesis in the context of anxiety. Some studies find no link (Saxe et al, 2006), while others observe an anxiogenic response associated with reduced neurogenesis (Bergami et al, 2009; Revest et al, 2009). Voluntary exercise leads to a robust increase in neurogenesis while also increasing anxiety-like behavior (Fuss et al, 2010b). However, it must also be noted that voluntary exercise can reduce anxiety (Duman et al, 2008; Salam et al, 2009), suggesting a variety of factors in addition to neurogenesis likely interact to modulate anxiety.

To address the relevance of neurogenesis to psychiatric illness, we have utilized a genetic approach in which hippocampal neurogenesis is reduced following deletion of ataxia telangeictasia-mutated and rad-3 related (ATR). ATR is necessary for genomic stability in the early embryo and cells lacking ATR ultimately exit the cell cycle or undergo programmed cell death (Brown and Baltimore, 2000, 2003). Ubiquitous deletion of ATR from the adult mouse leads to age-related phenotypes that are associated with the premature depletion of stem/progenitor cells (Ruzankina et al, 2007). Previous research has demonstrated that ATR can be deleted from differentiated forebrain CNS neurons by Synapsin1 promoter-driven Cre expression with no effects on circadian period, coordination, strength, anxiety, or hippocampal-dependent learning (Ruzankina et al, 2007). This suggests that ATR is not necessary for the function of differentiated neurons and implicates loss of ATR from the proliferating cell population in the behavioral consequences that we observe following hippocampalspecific deletion. Targeting a protein that is not necessary for the function of mature cells provides the added benefit of minimizing possible compensatory or nonspecific effects that could arise if the deleted protein were to have a role outside of cell division.

Here, we show that deletion of ATR from the adult hippocampus leads to at least a $50 \%$ reduction in neurogenesis. Behavioral consequences of partially inhibiting neurogenesis have been observed previously (Revest et al, 2009; Conboy et al, 2010). We thus evaluated behavior in rodent models of affective state and examined the cellular and behavioral effects of $\mathrm{AD}$ treatment following ATR deletion. Although not a complete ablation, this model has the potential to mimic the reduced rate of neurogenesis that is observed during aging (Bondolfi et al, 2004). Aged mice have a reduced capacity for AD-induced neurogenesis (Couillard-Despres et al, 2009) and treatment-resistant depression is prevalent in the aged population (Lenze et al, 2008).

\section{SUBJECTS AND METHODS}

See Supplementary Information for adeno-associated virus and microinjection methods.

\section{Animals}

Mice homozygous for the Cre/lox-conditional allele of ATR (referred to as floxed-ATR or ATR ${ }^{\mathrm{f} / \mathrm{f}}$ ) and mice expressing the tamoxifen-inducible Cre transgene $\left(\mathrm{CreERT}_{2}\right)$ on a 129 SV/C57BL/6 background were generated as previously described (Ruzankina et al, 2007). Floxed-ATR mice were used for hippocampal-specific ATR deletion. For wholebody deletion of ATR, floxed-ATR mice carrying CreERT ${ }_{2}$ were treated with tamoxifen as described (Ruzankina et al, 2007). For behavioral studies in these mice, one of two control groups was utilized: (1) $\mathrm{ATR}^{\mathrm{f} / \mathrm{f}} ; \mathrm{CreERT}_{2}+$ vehicle, or (2) $\mathrm{ATR}^{\mathrm{f} / \mathrm{f}}+$ tamoxifen. Mice (1.5-5 months old, 20$35 \mathrm{~g}$ ) were housed in groups of 3-4 following stereotaxic surgery and during experimental paradigms, with exceptions noted. All behavioral experiments were conducted on males and females at least 5 weeks following stereotaxic surgery or tamoxifen treatment. Animals were maintained on a $12 \mathrm{~h}$ light-dark cycle with food and water available $a d$ libitum in accordance with the University of Pennsylvania Institutional Animal Care and Use Committee. In some instances multiple behavioral tests were performed in a single cohort of mice, as indicated here: (1) elevated zero maze was performed 3 days following marble burying. (2) Training in the novelty-induced hypophagia (NIH) commenced 3 days following forced swim test. (3) Locomotor activity was measured 2 days before the Morris water maze and restraint stress was measured 1 week following water maze testing.

\section{Drugs}

DMI (Sigma, St Louis, MO), chlordiazepoxide (CDP, Sigma) and bromodeoxyuridine (BrdU, Sigma) were dissolved in $0.9 \%$ saline. Solutions were prepared immediately before use and injected intraperitoneally (i.p.) using a volume of $10 \mathrm{ml} / \mathrm{kg}$. For cell proliferation analysis, $200 \mathrm{mg} / \mathrm{kg} \mathrm{BrdU}$ was administered $24 \mathrm{~h}$ before perfusion. For cell survival analysis, $100 \mathrm{mg} / \mathrm{kg}$ BrdU was administered once per day for 4 days, 4 weeks before perfusion. To activate the inducible CreERT $_{2}$, tamoxifen (Sigma) was dissolved at a concentration of $20 \mathrm{mg} / \mathrm{ml}$ in a mixture of $98 \%$ corn oil per $2 \%$ ethanol and was injected i.p. once per day for 4 days.

\section{Quantitative-PCR}

Cre/Lox recombination was quantified using RT-PCR. Brains were rapidly removed and hippocampi were dissected out and flash-frozen in liquid nitrogen. DNA was extracted using Qiagen DNeasy Blood and Tissue Kit (Qiagen, Valencia, CA). Deletion rate was analyzed by utilizing a Taqman primer/probe set, which overlap the $3^{\prime}$ lox sequence and a portion of the excised domain as described (Ruzankina et al, 2009). Floxed-ATR levels were normalized to the housekeeping gene TBP.

\section{Immunohistochemistry}

Mice were anesthetized with nembutol $(10 \mathrm{mg} / \mathrm{kg})$ and transcardially perfused with cold $0.1 \mathrm{M}$ phosphate-buffered saline (PBS) for $5 \mathrm{~min}$, followed by cold $4 \%$ paraformaldehyde 
(PFA) in PBS for $15 \mathrm{~min}$. Brains were postfixed overnight in PFA at $4{ }^{\circ} \mathrm{C}$ and subsequently stored at $4{ }^{\circ} \mathrm{C}$ in $30 \%$ sucrose. Brains were frozen on dry ice, sectioned coronally at a thickness of $40 \mu \mathrm{m}$, and transferred to PBS + $0.5 \%$ sodium azide at $4{ }^{\circ} \mathrm{C}$ before processing for immunohistochemistry.

For BrdU analysis, sections were wet-mounted on Superfrost/Plus slides (Fisher Scientific, Pittsburgh, PA) for stereological analysis using a modified version of the optical fractionator method (West et al, 1991). Mounted sections were incubated in $0.1 \mathrm{M}$ citric acid, $\mathrm{pH}$ 6.0, for antigen retrieval. Subsequently, slides were incubated in trypsin, $2 \mathrm{~N}$ $\mathrm{HCl}$, then primary antibody (mouse anti-BrdU, 1:200, Becton Dickinson) with $0.5 \%$ Tween 20 overnight. On the second day, slides were incubated for $1 \mathrm{~h}$ in secondary antibody (biotinylated anti-mouse IgG, Vector Laboratories), $1 \mathrm{~h}$ in avidin-biotin-HRP (1:100, Vector Laboratories), and labeled cells were visualized using diaminobenzidine (Sigma). PBS-washed slides were then counterstained and coverslipped with Permount. BrdU-IR cells in every ninth section of the hippocampus were counted with a $100 \times$ oil immersion lens, omitting cells in the upper-most focal plane. Cells within clusters were counted by distinguishing nuclear borders while focusing down through the tissue using an objective with a narrow depth of focus. For proliferation counts, a cell is included in the subgranular zone (SGZ) of the dentate gyrus if it is touching, or within two cells of the granule cell layer (GCL).

For doublecortin (DCX) analysis, free-floating sections of a $1 / 9$ series through the hippocampus were blocked for $1 \mathrm{~h}$ with $3 \%$ normal horse serum, $0.5 \%$ Tween 20 and $0.2 \%$ Triton in PBS (blocking solution). Sections were then incubated for $72 \mathrm{~h}$ at $4{ }^{\circ} \mathrm{C}$ in primary antibody (goat antiDCX, Santa Cruz \#8066) diluted 1:500 in blocking solution. Sections were washed and incubated for $1 \mathrm{~h}$ in secondary antibody (horse anti-goat, Vector Laboratories) diluted $1: 200$ in blocking solution. Sections were then washed and treated with $0.75 \% \mathrm{H}_{2} \mathrm{O}_{2}$ for $20 \mathrm{~min}$ before incubation in avidin-biotin-HRP ( $1: 200$, Vector Laboratories). Labeled cells were visualized using nickel-enhanced diaminobenzidine. Free-floating sections were mounted onto slides and dried overnight before dehydrating and coverslipping with Permount. DCX-IR cells in every ninth section were counted with a $100 \times$ oil immersion lens, omitting cells in the upper-most focal plane. All cells located within the GCL and SGZ were counted.

\section{Golgi Impregnation}

Golgi impregnation was conducted to label neurons and their processes for analysis of dendritic spine density according to the FD Rapid GolgiStain Kit (FD NeuroTechnologies, Ellicott City, MD). In all, $100 \mu \mathrm{m}$ sections were cleared with xylenes and coverslipped with Permount mounting medium. Hippocampal CA1 neurons selected for analysis had the following characteristics: dark and complete impregnation, untruncated tertiary dendrites, and isolated cell body with a clear connection to dendrites that are isolated from neighboring cells to avoid interference. For each brain, five CA1 neurons were selected for analysis. Within each neuron, six separate $10 \mu \mathrm{m}$ segments of tertiary dendrite, located at least $50 \mu \mathrm{m}$ from the cell body, were selected for counting. Number of spines per $10 \mu \mathrm{m}$ was determined manually using a confocal microscope (Nikon Eclipse E600, Nikon) with a $100 \times$ oil immersion lens. For each treatment group, average spine density of each neuron analyzed was averaged to arrive at the spine density used for statistical analysis.

\section{Elevated Zero Maze}

The elevated zero maze test was performed as described previously (Gundersen and Blendy, 2009). The maze (Stoelting, Wood Dale, IL) consists of two open areas (wall height, $0.5^{\prime \prime}$ ) and two closed areas (wall height, $12^{\prime \prime}$ ), and is elevated $24^{\prime \prime}$ from the ground. Lighting in the maze was 15 lux. At the start of testing, mice were placed into one of the closed areas and were allowed to explore the maze for 300 s. The Viewpoint Tracking System (Viewpoint, Champagne au Mont d'Or, France) was used to track time spent in the open areas, number of entries into the open areas, and distance traveled in each area.

\section{Marble Burying}

After a 1-h acclimation period in the testing room, mice were placed in a standard mouse cage containing $5 \mathrm{~cm}$ of bedding and 20 marbles, spaced equally along the edge of the cage. A wire-mesh lid was placed on the cage. Number of marbles buried at the end of a 15-min session was quantified. A marble was considered buried if it was at least $2 / 3$ beneath the surface of the bedding.

\section{NIH Test}

The NIH test was performed as previously described (Gur et al, 2007). Briefly, mice were pair-housed and trained to consume peanut butter chips (Nestle) in their home cage. Drug treatment occurred between the training and testing periods. For chronic AD treatment, mice received 25 days of DMI (12.5 mg/kg DMI, b.i.d.). This was followed by measuring latency to consume the palatable food on two consecutive test days. The first test day took place in the home cage. On the second test day, latency was measured in a novel environment, which consisted of an empty cage with no bedding, placed in a white box with a bright light (2150 lux) and a novel smell (dilute pine sol). On home and novel test days, mice received their first daily injection of DMI $1 \mathrm{~h}$ before testing latency to feed, and received their second injection of DMI in the evening, consistent with the chronic treatment paradigm. For acute CDP treatment, home and novel test days occur immediately following the training period. CDP $(10 \mathrm{mg} / \mathrm{kg})$ was injected $30 \mathrm{~min}$ before the novel test.

\section{Forced Swim Test}

Mice were placed in water $\left(15 \mathrm{~cm}\right.$ depth, $\left.23^{\circ} \mathrm{C}\right)$ in plastic cylinders $(23 \mathrm{~cm}$ tall $\times 14 \mathrm{~cm}$ diameter $)$ for $6 \mathrm{~min}$. Using Viewpoint automated scoring (Viewpoint), duration of immobility was measured. Mice were exposed to the swim tanks twice (pre-swim and swim test), with $24 \mathrm{~h}$ between exposures. Three injections of DMI were given during the 
inter-swim interval: one $24 \mathrm{~h}$ before the swim test $(10 \mathrm{mg} / \mathrm{kg})$, a second injection $5 \mathrm{~h}$ before the swim test $(10 \mathrm{mg} / \mathrm{kg})$, and a third injection $1 \mathrm{~h}$ before the swim test $(20 \mathrm{mg} / \mathrm{kg})$.

\section{Statistical Analysis}

Statistical analyses were performed with GraphPad Prism 5. Data are reported as mean \pm SEM. Studies comparing AAV injection were analyzed with Student's $t$-tests. Studies comparing $\mathrm{AAV}$ and environment or treatment were analyzed by two-way ANOVA followed by pair-wise comparisons with Bonferroni post hoc tests.

\section{RESULTS}

\section{Hippocampal ATR Deletion Reduces Neurogenesis}

To delete ATR, we micro-injected AAV.Cre into both the dorsal and ventral hippocampus. AAV.Cre and the control virus, AAV.eGFP, were packaged into viral capsids of the serotype 9. AAV9 was chosen for its ability to spread throughout the hippocampus, thereby covering the entire rostral-caudal extent of the dentate gyrus without spreading outside of the hippocampal formation. Maximal deletion of ATR $\left(-78 \%, t_{(16)}=4.75, p<0.001\right)$ was evident 8 weeks following AAV injection (Figure 1a). Photomicrographs of eGFP fluorescence at this time point illustrate spread and expression of the AAV (Figures $1 \mathrm{~b}$ and $\mathrm{c}$ ).

To determine the effect of hippocampal ATR deletion on neurogenesis, floxed-ATR mice were injected i.p. with $200 \mathrm{mg} / \mathrm{kg}$ BrdU 8 weeks following hippocampal AAV microinjection and perfused $24 \mathrm{~h}$ later. Compared with controls, ATR-deleted mice showed significantly reduced $\left(-45 \%, t_{(17)}=5.56, p<0.0001\right) \mathrm{BrdU}$ incorporation, indicating a robust reduction in basal levels of cell proliferation (Figures 2a, d and e). To determine the effect of ATR deletion on cell survival, BrdU was injected 4 weeks following AAV microinjection and mice were perfused 4 weeks later. Compared with controls, ATR-deleted mice showed significantly reduced $\left(-57 \%, t_{(22)}=5.31\right.$, $p<0.0001) \mathrm{BrdU}$ incorporation, indicative of reduced cell survival (Figure 2b). Additionally, ATR-deleted mice showed significantly reduced $\left(-54 \%, t_{(17)}=3.246\right.$, $p<0.005)$ expression of DCX, indicating a reduction in the total number of newly-born, immature neurons present in the hippocampus (Figures $2 \mathrm{c}, \mathrm{f}$ and g). Taken together, these data demonstrate that hippocampal ATR deletion results in at least a 50\% reduction in neurogenesis. The requirement of ATR for normal rates of neurogenesis was verified in a second model of ATR deletion, $\mathrm{ATR}^{\mathrm{f} / \mathrm{f}} ; \mathrm{CreERT}_{2}$ mice treated with tamoxifen. Five weeks after tamoxifen

a

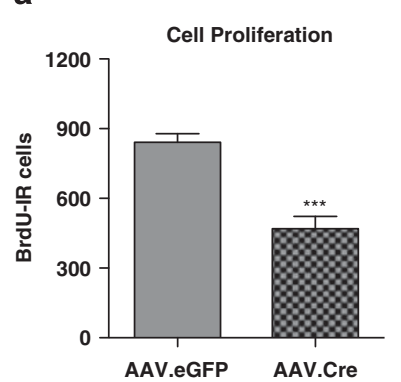

d

e

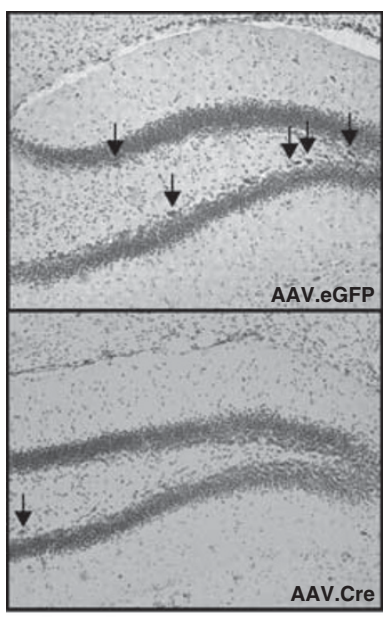

b Cell Survival

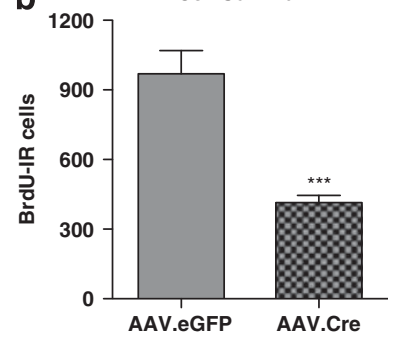

f

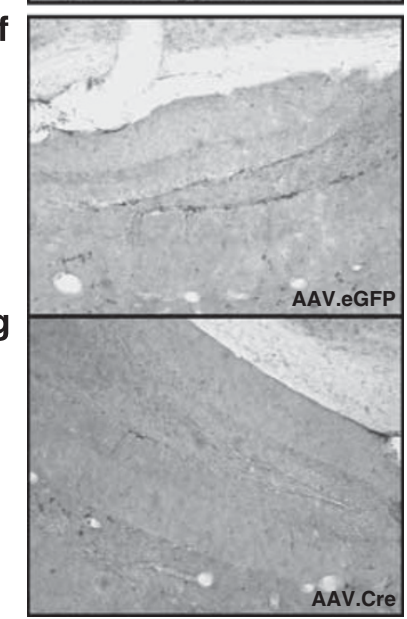

Figure 2 Hippocampal ATR deletion inhibits hippocampal neurogenesis. (a, b) AAV.Cre-injected mice exhibited significantly fewer BrdU-labeled cells within the subgranular zone as compared with AAV.eGFP-injected mice, both $24 \mathrm{~h}$ ( $a$, **** $p<0.000 \mathrm{I}, \mathrm{n}=9-10$ per group) and 4 weeks (b, ${ }^{*} * * * 0.000 I, n=12$ per group) after BrdU injection. (c) Using sections from the same mice as in Figure 2a, DCX quantification revealed a significant decrease in immature neurons in the dentate gyrus of AAV.Creinjected mice as compared with AAV.eGFP-injected mice (** $p<0.005$, $n=9-10$ per group). (d, e) Representative photomicrographs of immunostaining for BrdU. Arrows point to BrdU-IR cells. (f, g) Representative photomicrographs of immunostaining for DCX. Error bars indicate SEM.
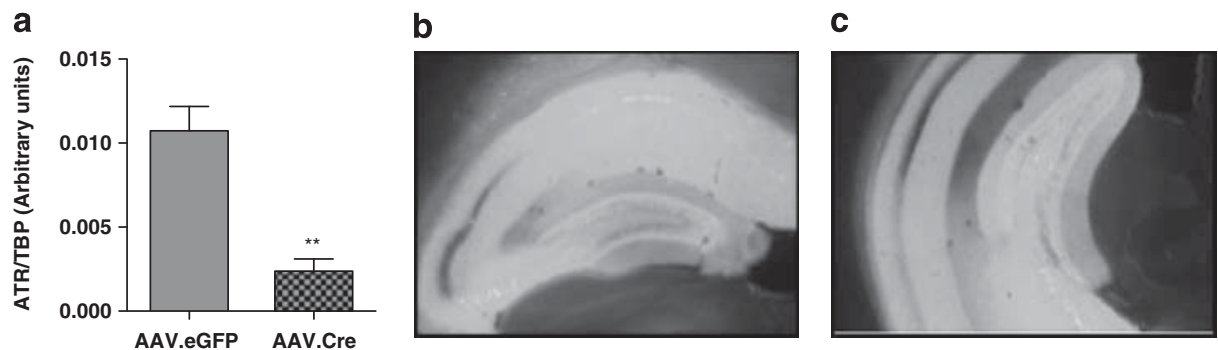

Figure I Hippocampal microinjection of AAV.Cre deletes ATR. (a) Floxed-ATR mice show a significant reduction in the floxed allele 8 weeks following hippocampal microinjection of AAV.Cre (** $p<0.001, n=7-9$ per group). (b, c) Expression pattern of eGFP in the dorsal (b) and ventral (c) hippocampus 8 weeks following hippocampal microinjection of AAV.eGFP. Error bars indicate SEM. 

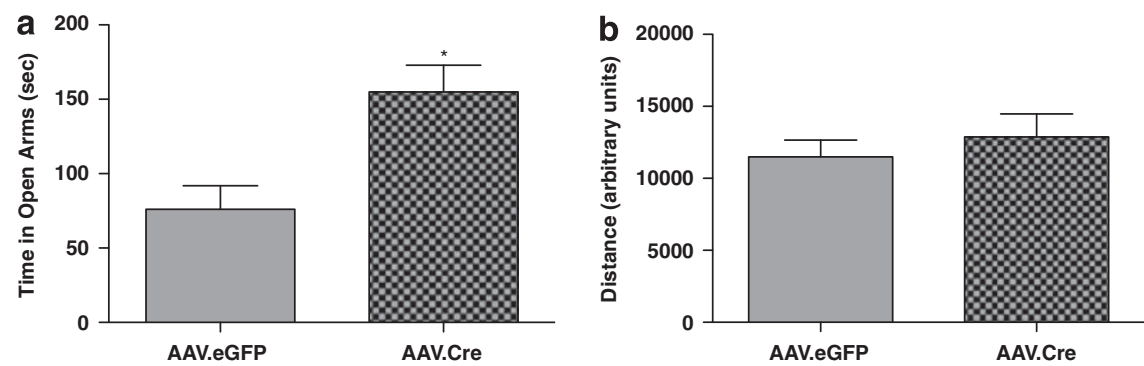

Figure 3 Hippocampal ATR deletion is associated with reduced anxiety in the elevated zero maze (EZM). (a) AAV.Cre-injected mice spent significantly more time in the open arms of the maze, as compared with AAV.eGFP-injected mice (* $p<0.01, n=5-7$ per group). (b) There was no difference between groups in the total distance travelled in the EZM. Error bars indicate SEM.
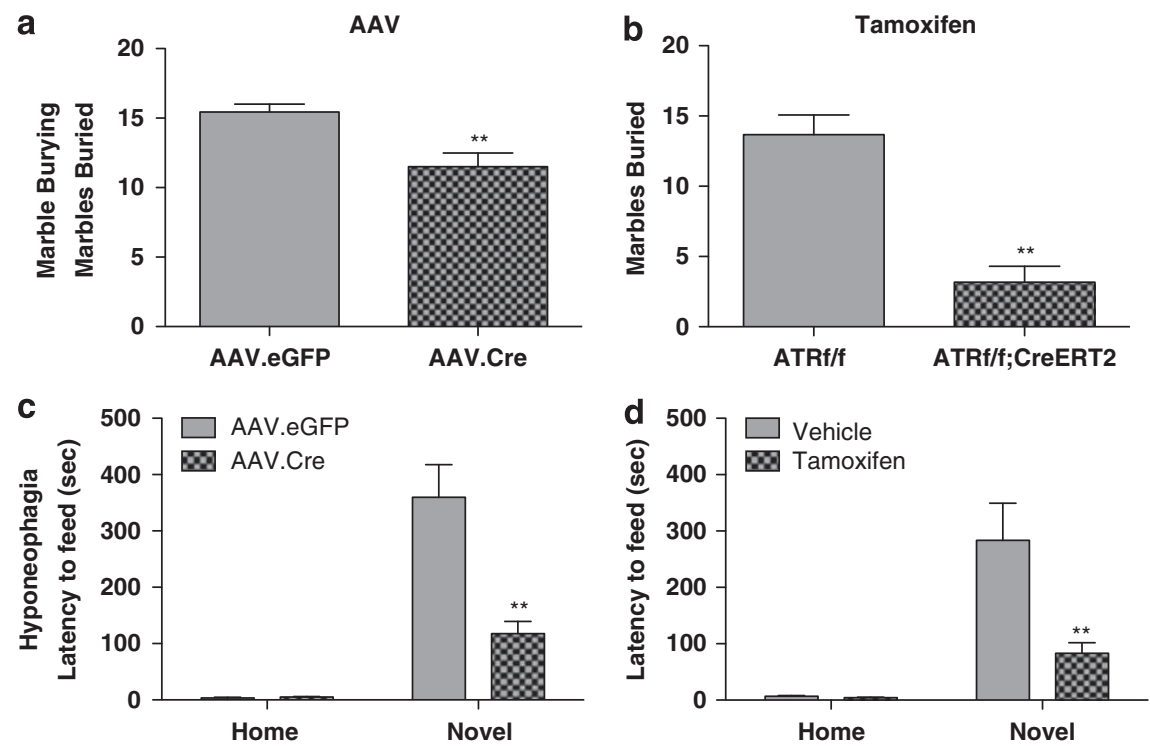

Figure 4 ATR deletion is associated with reduced anxiety. (a, b) ATR-deleted mice buried significantly fewer marbles during a I5-min marble burying test. This effect was observed in both hippocampal-specific ATR deletion ( $a$, *** $<<0.01, n=14-16$ per group) and in the tamoxifen-inducible ubiquitous ATR deletion ( $b$, *** $p<0.00 \mathrm{I}, n=6$ per group). (c, d) ATR-deleted mice exhibited significantly shorter latencies to feed in the novel environment during the $\mathrm{NIH}$ test. This effect was observed both in hippocampal-specific ATR deletion (c, ${ }^{*} * p<0.00$ I, $n=8$ per group) and in the tamoxifen-inducible ubiquitous ATR deletion (d, $* * * 0.001, n=11-13$ per group). Error bars indicate SEM.

treatment, we already observed a $30 \%$ reduction in BrdU incorporation in these mice ( $p=0.06$, data not shown).

\section{ATR Deletion Reduces Anxiety-Like Behavior}

After establishing that hippocampal ATR deletion results in reduced basal levels of neurogenesis, we sought to evaluate behavior of the floxed-ATR mice in rodent models of affective state. Anxiety-like behavior was evaluated using the EZM (Figure 3a). ATR-deleted mice spent significantly greater time $\left(t_{(10)}=3.26, p<0.01\right)$ in the open arms of the maze, indicating reduced levels of anxiety compared with controls. This observation was not because of any overall difference in distance travelled in the maze $\left(t_{(10)}=0.71\right.$ $p=0.49$ ) (Figure $3 \mathrm{~b}$ ). Furthermore, deletion of ATR from the hippocampus did not affect general locomotor activity or corticosterone response to an acute stressor (Supplementary Figure S1).

The anxiolytic phenotype observed in the EZM is at odds with work by others demonstrating an anxiogenic phenotype following reduced neurogenesis (Bergami et al, 2009; Revest et al, 2009). To determine if reduced anxiety-like behavior was present in other tests, we examined behavior in the marble burying test in both the AAV and tamoxifeninducible models of ATR deletion. As illustrated in Figure 4a, hippocampal ATR deletion was associated with a significant reduction $\left(t_{(28)}=3.55, p<0.01\right)$ in the number of marbles buried during a 15-min session. Consistent with the anxiolytic effect of hippocampal-specific ATR deletion, reduced anxiety-like behavior was also observed in the marble burying test in $\mathrm{ATR}^{\mathrm{f} / \mathrm{f}} ; \mathrm{CreERT}_{2}$ mice treated with tamoxifen $\left(t_{(10)}=5.805, p<0.05\right)$ (Figure $4 \mathrm{~b}$ ).

ATR deletion resulted in reduced hyponeophagia in the $\mathrm{NIH}$ test, indicative of a blunted anxiety-like response to a novel, anxiety-provoking environment. Mice were trained to consume peanut butter chips in their home cage and subsequently tested for latency to consume this palatable food in a novel, anxiety-provoking environment. Following hippocampal-specific deletion, ATR-deleted mice showed significantly shorter latency to feed in the novel environment compared with controls $(p<0.001)$ (Figure $4 c)$. Two-way ANOVA revealed significant main effects of environment $\left(\mathrm{F}_{(1,28)}=57.87, p<0.0001\right)$ and $\operatorname{AAV}\left(\mathrm{F}_{(1,28)}=15.23\right.$, $p<0.001)$, as well as a significant environment $\times \mathrm{AAV}$ 

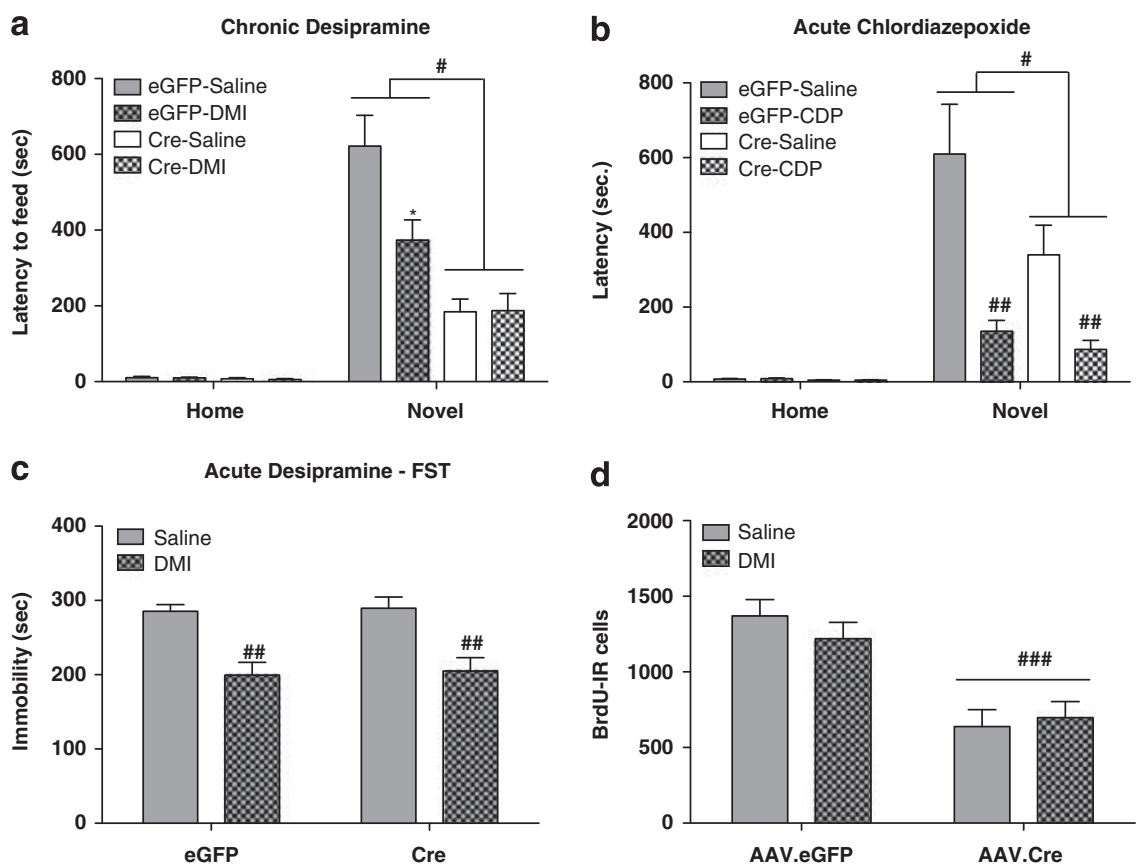

Figure 5 In the NIH paradigm, ATR-deleted mice exhibit altered antidepressant response. (a) In a novel environment, AAV.Cre-injected mice exhibited a reduction in latency to consume peanut butter chips compared with AAV.eGFP-injected mice $\left({ }^{*} p<0.000 \mathrm{I}, n=7-8\right.$ per group). There was a significant effect of chronic DMI treatment ( $12.5 \mathrm{mg} / \mathrm{kg}$ b.i.d., 24 days) only in the AAV.eGFP-injected mice (* $p<0.05)$. (b) CDP administered 30 min before novel testing reduced latency to feed in both control and ATR-deleted mice $\left({ }^{\# \#} p<0.000\right.$ I vs saline-treated, $n=8-10$ per group). (c) DMI significantly reduced immobility in the FST in both control and ATR-deleted mice (\# $p<0.0001$ vs saline-treated, $n=8-10$ per group). DMI was administered $24 \mathrm{~h}$ ( $10 \mathrm{mg} / \mathrm{kg}$ ), $5 \mathrm{~h}(10 \mathrm{mg} / \mathrm{kg})$, and I $\mathrm{h}(20 \mathrm{mg} / \mathrm{kg})$ before the 6-min swim test. Immobility was measured over the final $4 \mathrm{~min}$. (d) ATR deletion reduced cell proliferation in the dentate gyrus (\#\#\#<0.00I vs AAV.eGFP-treated, $n=7-8$ per group). Chronic DMI ( $12.5 \mathrm{mg} / \mathrm{kg}$ b.i.d., 24 days) had no effect on cell proliferation. Error bars indicate SEM.

interaction $\left(\mathrm{F}_{(1,28)}=15.65, p<0.001\right)$. Consistent with these findings, reduced anxiety was also observed in the $\mathrm{ATR}^{\mathrm{f} /}$ ${ }_{\text {; }}$ CreERT $_{2}$ mice in the NIH test (Figure 4d). Two-way ANOVA revealed significant main effects of environment $\left(\mathrm{F}_{(1,45)}=32.44\right.$, $p<0.0001)$ and genotype $\left(\mathrm{F}_{(1,45)}=10.61, p<0.005\right)$, as well as a significant environment $\times$ genotype interaction $\left(\mathrm{F}_{(1,45)}=10.08\right.$; $p<0.005)$. Post hoc analyses confirmed significantly reduced latency to feed $(p<0.001)$ in the novel environment in ATR-deleted mice compared with controls (Figure 4d).

\section{Behavioral Effects of DMI are Attenuated Following ATR Deletion}

As hippocampal neurogenesis has been implicated in the mechanism of action of ADs, we next sought to determine whether ATR deletion would alter the cellular and behavioral response to chronic $\mathrm{AD}$ treatment. To address this, we again utilized the NIH test but evaluated mice following chronic DMI treatment $(12.5 \mathrm{mg} / \mathrm{kg}$ i.p., b.i.d.). This dose effectively reduces feeding latency in mice on a 129SV/C57BL/6 background following chronic, but not acute treatment (Gur et al, 2007; Turner et al, 2010). All training occurred before drug exposure. Home cage testing occurred on the 24th day of DMI treatment and novel cage testing occurred on the 25th day. Following the novel cage test, mice were given $200 \mathrm{mg} / \mathrm{kg} \mathrm{BrdU}$ and perfused $24 \mathrm{~h}$ later. There were no effects of AAV or AD (AAV main effect: $\mathrm{F}_{(1,28)}=2.735, p=0.109 ; \mathrm{AD}$ main effect: $\mathrm{F}_{(1,28)}=0.02$, $p=0.889 ; \mathrm{AAV} \times \mathrm{AD}$ interaction: $\left.\mathrm{F}_{(1,28)}=0.003, p=0.957\right)$ on latency to feed in the home cage test (Figure 5a). Consistent with our finding that ATR deletion reduced hyponeophagia in the absence of drug treatment (Figure 4c), two-way ANOVA revealed a significant main effect of $\operatorname{AAV}\left(\mathrm{F}_{(1,25)}=23.40, p<0.0001\right)$ in the novel environment (Figure 5a). Additionally, there was a significant main effect of DMI $\left(\mathrm{F}_{(1,25)}=4.394, p<0.05\right)$ and a significant $\mathrm{AAV} \times \mathrm{AD}$ interaction $\left(\mathrm{F}_{(1,25)}=4.601, p<0.05\right)$ in the novel environment (Figure 5a). Post hoc analyses revealed a significant effect of DMI only in the AAV.eGFP treatment group $(p<0.05)$, indicating that DMI does not reduce hyponeophagia in ATR-deleted mice. This is unlikely because of a floor effect of this test, as acute treatment with CDP $(10 \mathrm{mg} / \mathrm{kg}) 30 \mathrm{~min}$ before the novel cage test resulted in significantly reduced latency to feed in the novel environment (Figure 5b) in both control and ATRdeleted mice (AAV main effect: $\mathrm{F}_{(1,24)}=4.863, p=<0.05$; CDP main effect: $\left.\mathrm{F}_{(1,24)}=25.45, p<0.0001\right)$. Additionally, ATR-deleted mice and controls responded equally well to DMI in the forced swim test (Figure $5 \mathrm{c}$ ) by demonstrating reduced immobility compared with saline-treated mice (DMI main effect: $\mathrm{F}_{(1,32)}=30.44, p<0.0001$ ). Furthermore, the altered behavior observed in the NIH is not likely because of learning or memory impairment, as ATR deletion did not induce deficits in associative (Supplementary Figure S2) or spatial learning (Supplementary Figure S3).

\section{Cellular Effects of Chronic DMI are Altered in ATR-Deleted Mice}

Following behavioral testing in the NIH paradigm, the cellular effects of chronic DMI treatment were evaluated. 
Two-way ANOVA revealed a significant main effect of AAV $\left(\mathrm{F}_{(1,29)}=31.73 ; p<0.0001\right)$ to reduce cell proliferation in ATR-deleted mice (Figure 5d), as observed previously. DMI had no effect on cell proliferation in control or ATR-deleted mice (AD main effect: $\mathrm{F}_{(1,29)}=0.173, p=0.68, \mathrm{AAV} \times \mathrm{AD}$ interaction: $\left.F_{(1,29)}=0.877, p=0.357\right)$. In the absence of a neurogenic effect of DMI treatment, we sought to identify a potential mechanism through which DMI may exert behavioral changes. Dendritic spine density, a correlate of synaptic transmission strength and neuronal plasticity (von Bohlen Und Halbach, 2009), can be increased by AD treatment in rodents (Norrholm and Ouimet, 2001; Hajszan et al, 2005). Reduced spine density has been observed in post-mortem tissue of depressed individuals (Law et al, 2004). To this end, we examined whether DMI exerted differential effects on CA1 dendritic spine density in ATRdeleted mice. Two-way ANOVA revealed significant main effects of $\operatorname{AAV}\left(\mathrm{F}_{(1,145)}=25.2, p<0.0001\right), \mathrm{AD}\left(\mathrm{F}_{(1,145)}=\right.$ 159.7, $p<0.0001)$, and a significant $\mathrm{AAV} \times \mathrm{AD}$ interaction $\left(\mathrm{F}_{(1,145)}=81.41, p<0.0001\right)$. Post hoc analyses confirmed a robust significant effect $(+34 \%, p<0.001)$ of DMI in control mice (Figures $6 \mathrm{a}$ and $\mathrm{b}$ ). This effect was greatly attenuated $(+5 \%, \quad p<0.05)$ in ATR-deleted mice (Figure 6c).

\section{DISCUSSION}

Since neurogenesis was first proposed to regulate response to $\mathrm{AD}$ treatment (Jacobs et al, 2000), numerous publications have examined hippocampal neurogenesis in the context of depression. However, its precise role in the etiology and treatment of mood disorders remains controversial. In this study, we showed that ATR is necessary for maintenance of the basal rate of hippocampal neurogenesis. Deletion of ATR from the hippocampus resulted in a partial inhibition of neurogenesis and reduced anxiety-like behavior in multiple mouse models of affective state. $\mathrm{AD}$ treatment was behaviorally ineffective in the NIH test following ATR deletion. In addition, the increased dendritic spine density induced by chronic treatment with DMI was severely attenuated following ATR deletion. These data suggest that capacity for increased neuronal plasticity in the hippocampus, rather than increased neurogenesis, may underlie the behavioral efficacy of DMI in the NIH test. A neurogenesis-independent effect of DMI in the NIH test is additionally supported by the observation that DMI reduced hyponeophagia in control mice without stimulating cell proliferation.

ATR is important for maintaining genomic integrity during DNA synthesis and proliferative failure is observed in its absence (Brown and Baltimore, 2000, 2003). No obvious behavioral changes are observed when ATR was deleted from differentiated cells in the forebrain (Ruzankina et al, 2007), suggesting that the behavioral alterations observed herein can be attributed to deletion of ATR from cells with proliferative potential following hippocampal AAV microinjection. The partial reduction in neurogenesis is likely related to the infection rate of the AAV in the neural stem and progenitor cells. Hypothetically, greater inhibition could be obtained by utilizing an approach that targets these cells with greater affinity. However, the significant
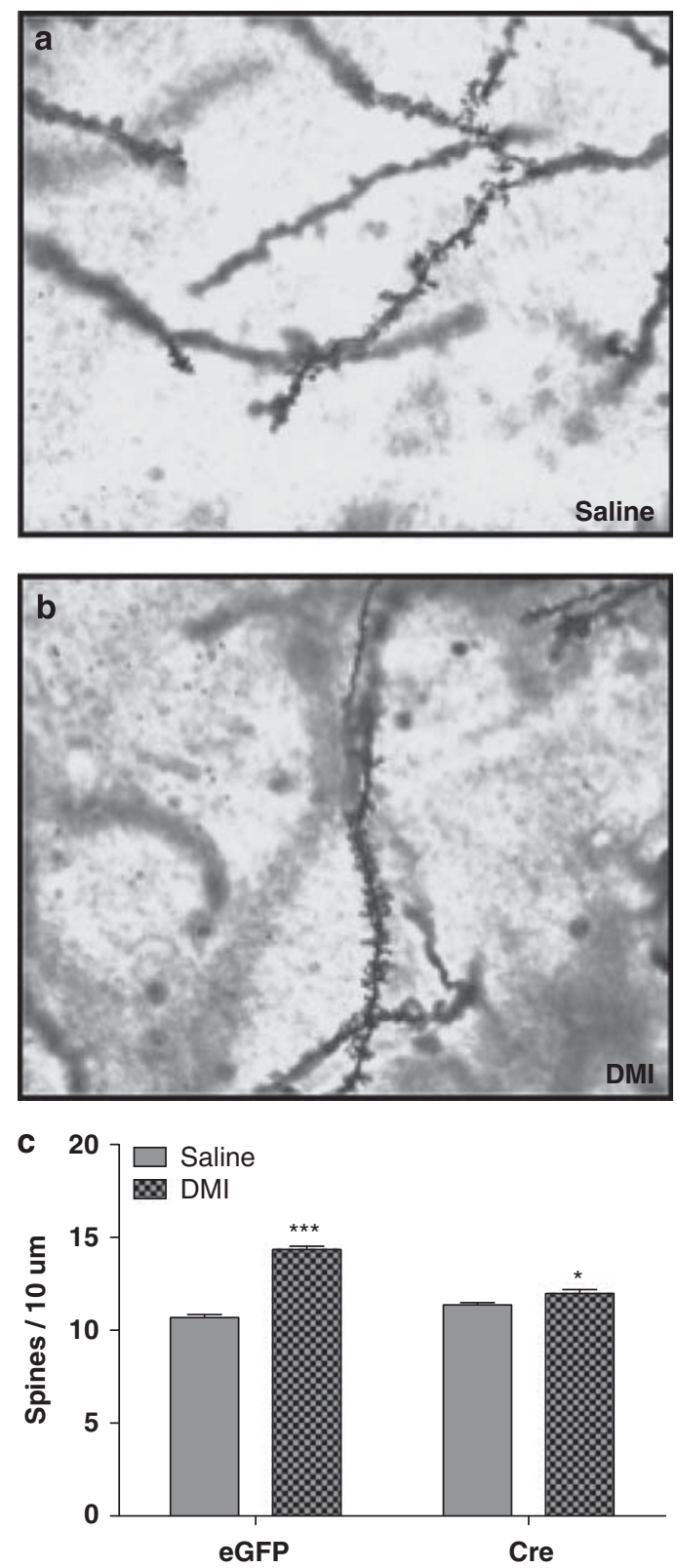

Figure 6 Hippocampal CAI dendritic spine density following DMI treatment. (a, b) Representative photomicrographs of Golgi impregnation staining to visualize dendritic arborization following saline (a) or DMI (b) treatment in AAV.eGFP-injected mice. (c) Chronic DMI treatment significantly increased CAI dendritic spine density in AAV.eGFP-injected mice (**** $p<0.00 \mathrm{I}, n=30-42$ per group). This effect was attenuated in AAV.Cre-injected mice. $(* p<0.05)$. Error bars indicate SEM.

cellular and behavioral effects observed in this model of partially-blocked neurogenesis reinforce the sensitivity of hippocampal functioning to changes in neurogenesis.

We observed a $45 \%$ reduction in hippocampal cell proliferation (Figure 2a) and a similar reduction in the total number of 4-week-old cells (Figure 2b), 8 weeks following ATR deletion. Consistent with this finding, 54\% fewer DCX-expressing cells were present in the hippocampus as a result of ATR deletion (Figure 2c). DCX is transiently expressed in immature neurons as they mature and migrate (Brown et al, 2003). These data indicate that 
ATR is necessary to maintain a normal rate of hippocampal neurogenesis. In a second model of ATR deletion that does not involve virus injection, we used a tamoxifen-inducible Cre transgene to delete ATR. In this model, we observed a $30 \%$ reduction in cell proliferation as early as 5 weeks after ATR deletion. Although this lesser reduction may be attributed to the abbreviated period between deletion and BrdU assay, the dose of tamoxifen and level of Cre induction may also be factors. Regardless, the reduced cell proliferation and reduced anxiety-like behavior observed in this second model used to delete ATR support our finding that ATR has a critical role in cell proliferation in the hippocampus.

ATR deletion resulted in reduced anxiety in multiple rodent behavioral tests, including the EZM (Figure 3), marble burying test (Figure $4 \mathrm{a}$ and $\mathrm{b}$ ), and NIH test (Figures $4 \mathrm{c}$ and $\mathrm{d}$ ). These observations are at odds with studies associating reduced neurogenesis with increased anxiety in the elevated plus maze (Revest et al, 2009; Conboy et al, 2010) and light-dark box (Scobie et al, 2009), and finding no baseline change in hyponeophagia (Santarelli et al, 2003; Revest et al, 2009; Scobie et al, 2009). Interestingly, recent literature (Fuss et al, 2010a) suggests that intermediate levels of neurogenesis are associated with low levels of anxiety in some paradigms, with exercise and irradiation, which increase and ablate neurogenesis, respectively, both associated with elevated anxiety. The anxiolytic phenotype observed in ATR-deleted mice, which exhibit levels of neurogenesis intermediate between basal levels and a complete ablation, appears to be in agreement with the aforementioned finding. Furthermore, at the opposite end of the spectrum, our data are in line with studies demonstrating a correlation between increased neurogenesis and increased anxiety following voluntary exercise (Fuss et al, 2010b). This association between increased neurogenesis and increased anxiety is also observed in CREB-deficient mice (Gur et al, 2007).

The NIH test is considered an ethologically relevant test with strong predictive and construct validity (Dulawa and Hen, 2005). Our lab and others have previously demonstrated that latency to feed in the novel environment can be reduced by acute treatment with a benzodiazepine, as well as treatment with chronic, but not acute, AD compounds (Dulawa and Hen, 2005, for review; Gur et al, 2007; Gundersen and Blendy, 2009). Our interest in examining the ATR mice in the NIH test was twofold. First, baseline behavior in the novel environment provides a measure of anxiety. Second, this behavior is sensitive to chronic AD treatment that is thought to depend on increased neurogenesis. However, in studies where X-ray irradiation is used to ablate neurogenesis, there are discrepancies in the resulting effects on both baseline and AD-mediated hyponeophagia (Santarelli et al, 2003; Holick et al, 2008; David et al, 2009). These conflicting findings may stem from variations in mouse strain, AD treatment, or hyponeophagia protocol, as some investigators utilize the novelty-suppressed feeding test, a variation of the NIH test that involves food restriction. Additionally, reported effects of X-ray irradiation on differentiation (Tada et al, 2000) and inflammatory response (Mizumatsu et al, 2003) have the potential to interfere with evaluation of hippocampalmodulated behaviors. Whether or not these factors confound the findings of studies using X-ray irradiation, confirmation by genetic methods in which neurogenesis is reduced or ablated will lend confidence to the interpretations and implications of altered neurogenesis and $\mathrm{AD}$ responsivity. To date, no one has reported whether a partial deficit in neurogenesis generated by genetic manipulation, such as that seen following ATR deletion, alters AD efficacy in a chronic exposure paradigm.

We observed a significant behavioral effect of DMI treatment in the NIH test only in our control mice (Figure 5a). As baseline latencies were lower in the ATRdeleted mice, we ruled out a floor effect by testing the efficacy of a benzodiazepine, CDP, on latency in the novel test following ATR deletion. CDP significantly reduced latency to feed following ATR deletion (Figure 5b). Both control and ATR-deleted mice exhibited reduced immobility time in the forced swim test after acute treatment (Figure 5c), ruling out a more general deficit in response to DMI following ATR deletion.

On the basis of the behavioral observations in the $\mathrm{NIH}$ test, we hypothesized that DMI treatment in AAV.eGFPinjected mice would lead to increased neurogenesis, and that this effect of DMI would be attenuated in ATR-deleted mice. Interestingly, we observed no effect of DMI treatment on cell proliferation in either the control or ATR-deleted mice (Figure 5d). Although this was an unexpected observation, previous work has established that AD effects on neurogenesis can be small in magnitude and depend on numerous factors including mouse strain and pretreatment (Balu et al, 2009; David et al, 2009). Furthermore, others have reported no effects of AD treatment on cell proliferation in rodents (Huang et al, 2008; Navailles et al, 2008; Petersen et al, 2009).

DMI reduced latency to feed without affecting cell proliferation, indicating that DMI's effect on latency is through a neurogenesis-independent mechanism. To better understand this dissociation between DMI's effects on neurogenesis and behavior in the NIH paradigm, we sought to determine whether DMI exerts other trophic effects in the hippocampus, and whether those effects may explain the lack of efficacy of DMI in the NIH paradigm following ATR deletion. To this end, we observed significantly-increased CA1 dendritic spine density in control mice treated with DMI; this effect was greatly attenuated following ATR deletion (Figure 6c). Dendritic spine density is enhanced by various external stimuli including learning (Moser et al, 1994), enrichment (Zhu et al, 2009), exercise (Stranahan et al, 2009), and $\mathrm{AD}$ treatment; all stimuli that can have a positive influence on mood and emotional affect in depressed individuals. In agreement with work by others (Bessa et al, 2009), our results suggest that neuronal plasticity and/or remodeling, not increased neurogenesis, underlies the behavioral efficacy of AD treatment.

As ATR is not necessary for the functioning of mature cells, attenuation of DMI-mediated increases in dendritic spine density in the CA1 region, an area of the hippocampus composed of mature neurons, hints at broad, circuit-wide changes in hippocampal function as a result of reduced neurogenesis. Immature neurons in the adult hippocampus exhibit unique properties including enhanced excitability and a lower threshold for the induction of long-term potentiation, indicative of enhanced potential for synaptic 
plasticity (Schmidt-Hieber et al, 2004). A reduction in the proportion of hippocampal neurons, which exhibit these unique properties, as well a reduction in the number of new neurons available to functionally integrate into the hippocampal circuitry, may interfere with homeostatic functioning of the hippocampus, indirectly rendering it less responsive to the changes normally induced by DMI. Consistent with this hypothesis, ablation of neurogenesis leads to a reduction in evoked perforant path responses and an increase in the amplitude of spontaneous network activity in the dentate gyrus in an in vivo model (Lacefield et al, 2010). It is not unreasonable to predict that changes in network activity in the dentate gyrus because of loss of young, plastic neurons might extend to interrupting circuitry function within other regions of the hippocampus. The importance of neurogenesis for the treatment of mood disorders may thus lie in the role of new neurons in maintaining homeostatic network activity within the hippocampus, and this concept is supported by evidence that FLX depends on the plasticity of young neurons for its anxiolytic and AD efficacy (Wang et al, 2008).

Hippocampal ATR deletion provides a valuable model for studying the behavioral changes associated with deficits in hippocampal neurogenesis. In future studies, this model will be a useful tool for determining whether different classes of $\mathrm{AD}$ compounds exhibit varying degrees of neurogenesis dependence. In addition, it is of interest to evaluate susceptibility to depressive-like behaviors in mouse models of depression such as chronic mild stress and chronic social defeat in the ATR-deleted mice. Further insight into the pathophysiology and treatment of depression should lead to the eventual development of more rapid-acting treatments with fewer undesired side effects.

\section{ACKNOWLEDGEMENTS}

This research was supported by NIH Grant DA-011649 and CA-143187. JL Onksen was supported by a pre-doctoral NRSA (MH-087103).

\section{DISCLOSURE}

The authors declare no conflict of interest.

\section{REFERENCES}

Balu DT, Hodes GE, Anderson BT, Lucki I (2009). Enhanced sensitivity of the MRL/MpJ mouse to the neuroplastic and behavioral effects of chronic antidepressant treatments. Neuropsychopharmacology 34: 1764-1773.

Balu DT, Lucki I (2009). Adult hippocampal neurogenesis: regulation, functional implications, and contribution to disease pathology. Neurosci Biobehav Rev 33: 232-252.

Bergami M, Berninger B, Canossa M (2009). Conditional deletion of TrkB alters adult hippocampal neurogenesis and anxietyrelated behavior. Commun Integr Biol 2: 14-16.

Bessa JM, Ferreira D, Melo I, Marques F, Cerqueira JJ, Palha JA et al (2009). The mood-improving actions of antidepressants do not depend on neurogenesis but are associated with neuronal remodeling. Mol Psychiatry 14: 764-773, 739.

Bondolfi L, Ermini F, Long JM, Ingram DK, Jucker M (2004). Impact of age and caloric restriction on neurogenesis in the dentate gyrus of C57BL/6 mice. Neurobiol Aging 25: 333-340.
Brown EJ, Baltimore D (2000). ATR disruption leads to chromosomal fragmentation and early embryonic lethality. Genes Dev 14: 397-402.

Brown EJ, Baltimore D (2003). Essential and dispensable roles of ATR in cell cycle arrest and genome maintenance. Genes Dev 17: 615-628.

Brown JP, Couillard-Despres S, Cooper-Kuhn CM, Winkler J, Aigner L, Kuhn HG (2003). Transient expression of doublecortin during adult neurogenesis. J Comp Neurol 467: 1-10.

Calabrese F, Molteni R, Racagni G, Riva MA (2009). Neuronal plasticity: a link between stress and mood disorders. Psychoneuroendocrinology 34(Suppl 1): S208-S216.

Conboy L, Varea E, Castro JE, Sakouhi-Ouertatani H, Calandra T, Lashuel HA et al (2010). Macrophage migration inhibitory factor is critically involved in basal and fluoxetine-stimulated adult hippocampal cell proliferation and in anxiety, depression, and memory-related behaviors. Mol Psychiatry (e-pub ahead of print 23 February 2010).

Couillard-Despres S, Wuertinger C, Kandasamy M, Caioni M, Stadler K, Aigner R et al (2009). Ageing abolishes the effects of fluoxetine on neurogenesis. Mol Psychiatry 14: 856-864.

David DJ, Klemenhagen KC, Holick KA, Saxe MD, Mendez I, Santarelli L et al (2007). Efficacy of the MCHR1 antagonist $\mathrm{N}$-[3-(1-\{[4-(3,4-difluorophenoxy)phenyl]methyl\}(4-piperidyl))-4methylphen yl]-2-methylpropanamide (SNAP 94847) in mouse models of anxiety and depression following acute and chronic administration is independent of hippocampal neurogenesis. J Pharmacol Exp Ther 321: 237-248.

David DJ, Samuels BA, Rainer Q, Wang JW, Marsteller D, Mendez I et al (2009). Neurogenesis-dependent and -independent effects of fluoxetine in an animal model of anxiety/depression. Neuron 62: 479-493.

Dulawa SC, Hen R (2005). Recent advances in animal models of chronic antidepressant effects: the novelty-induced hypophagia test. Neurosci Biobehav Rev 29: 771-783.

Duman CH, Schlesinger L, Russell DS, Duman RS (2008). Voluntary exercise produces antidepressant and anxiolytic behavioral effects in mice. Brain Res 1199: 148-158.

Elizalde N, Garcia-Garcia AL, Totterdell S, Gendive N, Venzala E, Ramirez MJ et al (2010). Sustained stress-induced changes in mice as a model for chronic depression. Psychopharmacology (Berl) 210: 393-406.

Fuss J, Ben Abdallah NM, Hensley FW, Weber KJ, Hellweg R, Gass P (2010a). Deletion of running-induced hippocampal neurogenesis by irradiation prevents development of an anxious phenotype in mice. PLoS One 5(9): e12769.

Fuss J, Ben Abdallah NM, Vogt MA, Touma C, Pacifici PG, Palme R et al (2010b). Voluntary exercise induces anxiety-like behavior in adult C57BL/6J mice correlating with hippocampal neurogenesis. Hippocampus 20: 364-376.

Gundersen BB, Blendy JA (2009). Effects of the histone deacetylase inhibitor sodium butyrate in models of depression and anxiety. Neuropharmacology 57: 67-74.

Gur TL, Conti AC, Holden J, Bechtholt AJ, Hill TE, Lucki I et al (2007). cAMP response element-binding protein deficiency allows for increased neurogenesis and a rapid onset of antidepressant response. J Neurosci 27: 7860-7868.

Hajszan T, MacLusky NJ, Leranth C (2005). Short-term treatment with the antidepressant fluoxetine triggers pyramidal dendritic spine synapse formation in rat hippocampus. Eur J Neurosci 21: 1299-1303.

Holick KA, Lee DC, Hen R, Dulawa SC (2008). Behavioral effects of chronic fluoxetine in $\mathrm{BALB} / \mathrm{cJ}$ mice do not require adult hippocampal neurogenesis or the serotonin $1 \mathrm{~A}$ receptor. Neuropsychopharmacology 33: 406-417.

Huang GJ, Bannerman D, Flint J (2008). Chronic fluoxetine treatment alters behavior, but not adult hippocampal neurogenesis, in BALB/cJ mice. Mol Psychiatry 13: 119-121. 
Jacobs BL, van Praag H, Gage FH (2000). Adult brain neurogenesis and psychiatry: a novel theory of depression. Mol Psychiatry 5: 262-269.

Jessberger S, Kempermann G (2003). Adult-born hippocampal neurons mature into activity-dependent responsiveness. Eur J Neurosci 18: 2707-2712.

Lacefield CO, Itskov V, Reardon T, Hen R, Gordon JA (2010). Effects of adult-generated granule cells on coordinated network activity in the dentate gyrus. Hippocampus (e-pub ahead of print 29 September 2010, doi:10.1002/hipo.20860).

Law AJ, Weickert CS, Hyde TM, Kleinman JE, Harrison PJ (2004). Reduced spinophilin but not microtubule-associated protein 2 expression in the hippocampal formation in schizophrenia and mood disorders: molecular evidence for a pathology of dendritic spines. Am J Psychiatry 161: 1848-1855.

Lenze EJ, Sheffrin M, Driscoll HC, Mulsant BH, Pollock BG, Dew MA et al (2008). Incomplete response in late-life depression: getting to remission. Dialogues Clin Neurosci 10: 419-430.

Malberg JE, Duman RS (2003). Cell proliferation in adult hippocampus is decreased by inescapable stress: reversal by fluoxetine treatment. Neuropsychopharmacology 28: 1562-1571.

Malberg JE, Eisch AJ, Nestler EJ, Duman RS (2000). Chronic antidepressant treatment increases neurogenesis in adult rat hippocampus. J Neurosci 20: 9104-9110.

Marchetti C, Tafi E, Middei S, Rubinacci MA, Restivo L, Ammassari-Teule $M$ et al (2010). Synaptic adaptations of CA1 pyramidal neurons induced by a highly effective combinational antidepressant therapy. Biol Psychiatry 67: 146-154.

Merikangas KR, Zhang H, Avenevoli S, Acharyya S, Neuenschwander M, Angst J (2003). Longitudinal trajectories of depression and anxiety in a prospective community study: the Zurich Cohort Study. Arch Gen Psychiatry 60: 993-1000.

Mizumatsu S, Monje ML, Morhardt DR, Rola R, Palmer TD, Fike JR (2003). Extreme sensitivity of adult neurogenesis to low doses of X-irradiation. Cancer Res 63: 4021-4027.

Moser MB, Trommald M, Andersen P (1994). An increase in dendritic spine density on hippocampal CA1 pyramidal cells following spatial learning in adult rats suggests the formation of new synapses. Proc Natl Acad Sci USA 91: 12673-12675.

Murray F, Smith DW, Hutson PH (2008). Chronic low dose corticosterone exposure decreased hippocampal cell proliferation, volume and induced anxiety and depression like behaviours in mice. Eur J Pharmacol 583: 115-127.

Navailles S, Hof PR, Schmauss C (2008). Antidepressant druginduced stimulation of mouse hippocampal neurogenesis is agedependent and altered by early life stress. J Comp Neurol 509: 372-381.

Norrholm SD, Ouimet CC (2001). Altered dendritic spine density in animal models of depression and in response to antidepressant treatment. Synapse 42: 151-163.

O'Leary OF, Wu X, Castren E (2009). Chronic fluoxetine treatment increases expression of synaptic proteins in the hippocampus of the ovariectomized rat: role of BDNF signalling. Psychoneuroendocrinology 34: 367-381.

Papp M, Willner P, Muscat R (1991). An animal model of anhedonia: attenuation of sucrose consumption and place preference conditioning by chronic unpredictable mild stress. Psychopharmacology (Berl) 104: 255-259.

Petersen A, Wortwein G, Gruber SH, El-Khoury A, Mathe AA (2009). Nortriptyline mediates behavioral effects without affecting hippocampal cytogenesis in a genetic rat depression model. Neurosci Lett 451: 148-151.

Revest JM, Dupret D, Koehl M, Funk-Reiter C, Grosjean N, Piazza PV et al (2009). Adult hippocampal neurogenesis is involved in anxiety-related behaviors. Mol Psychiatry 14: 959-967.

Ruzankina Y, Pinzon-Guzman C, Asare A, Ong T, Pontano L, Cotsarelis G et al (2007). Deletion of the developmentally essential gene ATR in adult mice leads to age-related phenotypes and stem cell loss. Cell Stem Cell 1: 113-126.

Ruzankina Y, Schoppy DW, Asare A, Clark CE, Vonderheide RH, Brown EJ (2009). Tissue regenerative delays and synthetic lethality in adult mice after combined deletion of Atr and Trp53. Nat Genet 41: 1144-1149.

Sahay A, Hen R (2007). Adult hippocampal neurogenesis in depression. Nat Neurosci 10: 1110-1115.

Salam JN, Fox JH, Detroy EM, Guignon MH, Wohl DF, Falls WA (2009). Voluntary exercise in C57 mice is anxiolytic across several measures of anxiety. Behav Brain Res 197: 31-40.

Santarelli L, Saxe M, Gross C, Surget A, Battaglia F, Dulawa S et al (2003). Requirement of hippocampal neurogenesis for the behavioral effects of antidepressants. Science 301: 805-809.

Saxe MD, Battaglia F, Wang JW, Malleret G, David DJ, Monckton JE et al (2006). Ablation of hippocampal neurogenesis impairs contextual fear conditioning and synaptic plasticity in the dentate gyrus. Proc Natl Acad Sci USA 103: 17501-17506.

Schmidt-Hieber C, Jonas P, Bischofberger J (2004). Enhanced synaptic plasticity in newly generated granule cells of the adult hippocampus. Nature 429: 184-187.

Schoevers RA, Van HL, Koppelmans V, Kool S, Dekker JJ (2008). Managing the patient with co-morbid depression and an anxiety disorder. Drugs 68: 1621-1634.

Scobie KN, Hall BJ, Wilke SA, Klemenhagen KC, Fujii-Kuriyama Y, Ghosh A et al (2009). Kruppel-like factor 9 is necessary for latephase neuronal maturation in the developing dentate gyrus and during adult hippocampal neurogenesis. $J$ Neurosci 29: 9875-9887.

Stranahan AM, Lee K, Martin B, Maudsley S, Golden E, Cutler RG et al (2009). Voluntary exercise and caloric restriction enhance hippocampal dendritic spine density and BDNF levels in diabetic mice. Hippocampus 19: 951-961.

Tada E, Parent JM, Lowenstein DH, Fike JR (2000). X-irradiation causes a prolonged reduction in cell proliferation in the dentate gyrus of adult rats. Neuroscience 99: 33-41.

Turner JR, Castellano LM, Blendy JA (2010). Nicotinic partial agonists varenicline and sazetidine-A have differential effects on affective behavior. J Pharmacol Exp Ther 334: 665-672.

von Bohlen Und Halbach O (2009). Structure and function of dendritic spines within the hippocampus. Ann Anat 191: 518-531.

Wang JW, David DJ, Monckton JE, Battaglia F, Hen R (2008). Chronic fluoxetine stimulates maturation and synaptic plasticity of adult-born hippocampal granule cells. J Neurosci 28: 1374-1384.

West MJ, Slomianka L, Gundersen HJ (1991). Unbiased stereological estimation of the total number of neurons in thesubdivisions of the rat hippocampus using the optical fractionator. Anat Rec 231: 482-497.

Zhu SW, Codita A, Bogdanovic N, Hjerling-Leffler J, Ernfors P, Winblad B et al (2009). Influence of environmental manipulation on exploratory behaviour in male BDNF knockout mice. Behav Brain Res 197: 339-346.

Supplementary Information accompanies the paper on the Neuropsychopharmacology website (http://www.nature.com/npp) 